\title{
CAPSULE COMMENTARIES \\ Capsule Commentary on Michaelidis et al., Cost-Effectiveness of Procalcitonin-Guided Antibiotic Therapy for Outpatient Management of Acute Respiratory Tract Infections in Adults
}

\author{
Mary Lynn Davis-Ajami, PhD, MBA, MS, NP-C, RN \\ Organizational Systems and Adult Health, University of Maryland School of Nursing, Baltimore, MD, USA.
}

J Gen Intern Med 29(4):646

DOI: $10.1007 / \mathrm{s} 11606-013-2735-3$

(c) Society of General Internal Medicine 2013

\begin{abstract}
A ntibiotic overuse in ambulatory settings is a major public health concern. Acute respiratory infections are often treated empirically and present challenges to judicious antimicrobial use. Procalcitonin has emerged as a biomarker to discriminate between viral and bacterial respiratory infections, and may add value to standard decision guides. ${ }^{1}$ Acute respiratory infections are costly, and innovations minimizing drug or utilization costs would hold interest, particularly if cost-effective. One study ${ }^{2}$ examining antibiotic decision making using procalcitonin algorithms in the ICU found procalcitonin cost-effective when daily antibiotics cost more than $\$ 149(\mathrm{CAN})$, while another reports procalcitonin protocols costing more than usual care, with physician non-adherence to procalcitonin algorithms influencing cost-effectiveness in community acquired pneumonia. $^{3}$

Michaelidis et al. use a decision model with a health system perspective to estimate willingness-to-pay and costs per antibiotic prescription safely avoided for procalcitonin testing compared to usual care in adults with acute respiratory tract infections treated in outpatient settings. ${ }^{4}$ Overall, their results show that procalcitonin testing costs more than usual care, but reduces the number of antibiotic prescriptions. However, when including societal costs of antibiotic resistance and requirements for antimicrobial treatment, procalcitonin testing may be cost-effective.

Decision makers need to evaluate how well innovations maximize health outcomes within budgetary constraints to allocate resources. Although debate continues about costeffectiveness thresholds, ${ }^{5}$ knowledge diffusion, adoption and sustainable change management strategies may confound results when implementing innovative approaches such as procalcitonin testing. Michaelidis et al.'s decision model's bias towards procalcitonin testing assumes strong
\end{abstract}

Published online December 20, 2013 uptake of this innovation, and may overestimate availability while also omitting the impact of physician adherence on costs or cost-effectiveness. ${ }^{3}$ The cost-effectiveness model uses two European clinical trials, which may diminish generalizability. Although this study includes important secondary costs associated with antibiotic resistance, future research could further delineate appropriate antibiotic prescribing, include costs associated with antibiotic-induced adverse events, or compare procalcitonin protocol costs to antibiotic stewardship programs. ${ }^{1}$ Michaelidis et al.'s study brings important costeffectiveness information about outpatient procalcitonin testing in patients with acute respiratory infections, suggesting it is cost-effective when accounting for antibiotic resistance, although overestimating access to testing and underestimating physician non-adherence may diminish the benefit.

Conflict of Interest: The author has no conflicts of interest to report.

Corresponding Author: Mary Lynn Davis-Ajami, PhD, MBA, MS, NP-C, RN; Organizational Systems and Adult Health, University of Maryland School of Nursing, 655 West Lombard Street, 465D, Baltimore, MD 21201, USA (e-mail: davis-ajami@son.umaryland.edu).

\section{REFERENCES}

1. Schuetz P, Amin DN, Greenwald JL. Role of procalcitonin in managing adult patients with respiratory tract infections. Chest. 2012;141(4):106373. doi:10.1378/chet.11-2430.

2. Heyland DK, Johnson AP, Reynolds SC, Muscedere J. Procalcitonin for reduced antibiotic exposure in the critical care setting; a systematic review and an economic evaluation. Crit Care Med. 2011;39(7):179299.

3. Smith KJ, Wateska A, Nowalk MP, et al. Cost-effectiveness of procalcitonin-guided antibiotic use in community acquired pneumonia. J Gen Intern Med. 2013;28(9):1157-64. doi:10.1007/s11606-03-2400-x.

4. Michaelidis CI Zimmerman RK, Nowalk MP, et al. Cost-effectiveness of procalcitonin-guided antibiotic therapy for outpatient management of acute respiratory tract infections in adults. J Gen Int Med. 2013. doi:10.1007/s11606-013-2679-7.

5. Eichler H, Kong SX, Gerth WC, Mavros P, Jönsson B. Use of costeffectiveness analysis in health-care resource allocation decision-making: how are cost-effectiveness thresholds expected to emerge. Value Health. 2004;7(5):518-28. 